\title{
A importância dos hábitos alimentares saudáveis na terceira idade
}

The importance of healthy eating habits in old age

La importancia de los hábitos alimenticios saludables en la vejez

Recebido: 15/10/2021 | Revisado: 24/10/2021 | Aceito: 28/10/2021 | Publicado: 31/10/2021

\author{
Marcley Torres Ipuchima \\ ORCID: https://orcid.org/0000-0002-8413-4158 \\ Centro Universitário Nilton Lins, Brasil \\ E-mail: marcleyipuchima@gmail.com \\ Lídia Lisboa Costa \\ ORCID: https://orcid.org/0000-0003-4842-9618 \\ Centro Universitário Nilton Lins, Brasil \\ E-mail: lidialisboa7@gmail.com
}

\begin{abstract}
Resumo
O objetivo geral do estudo é destacar a importância dos hábitos alimentares saudáveis entre idosos desnutridos. Menciona-se que os objetivos específicos do estudo são: compreender a relação entre as alterações fisiológicas que acontecem no envelhecimento e as necessidades nutricionais do idoso; levantar aspectos da etiologia da desnutrição em idosos; e destacar a importância do acompanhamento nutricional na população idosa no desenvolvimento de hábitos saudáveis. Esta pesquisa trata-se de uma revisão da integrativa. A base de dados utilizadas foram: PubMed, Biblioteca Virtual de Saúde (BVS) e SciELO e outras revistas de nutrição. A estratégia elaborada para a inclusão nessa pesquisa foram: estudo publicados em revistas indexadas em inglês, espanhol e português, disponíveis na íntegra, gratuitos, que abordassem sobre o tema proposto, que foram devidamente publicados no período de 2015 a 2021. A estratégia elaborada para exclusão foram estudos publicados antes de 2015, que não estavam disponíveis na integra, e não tinham relação com o tema proposto. Assim, foram encontrados 367 estudos, desse total foram selecionados 85 , mas apenas 37 estudos foram analisados nesse artigo. Conclui-se que existem diferentes fatores de risco para a desnutrição em idosos, esse estado nutricional influência o desenvolvimento de doenças, podendo levá-lo até a morte. Por isso, familiares devem atuar para evitar tal quadro, mas quando desenvolvidos é relevante que o idoso seja acompanhado pelo nutricionista, pois o profissional pode analisar o quadro clínico e desenvolver estratégias alimentares adequadas aos pacientes.
\end{abstract}

Palavras-chave: Qualidade de vida; Hábitos alimentares; Pessoa idosa.

\begin{abstract}
The overall objective of the study is to highlight the importance of healthy eating habits among malnourished elderly. It is mentioned that the specific objectives of the study are: To understand the relationship between the physiological changes that occur in aging and the nutritional needs of the elderly. Raise aspects of the etiology of malnutrition in the elderly; and highlight the importance of nutritional follow-up in the elderly population in the development of healthy habits. This research is a review of the integrative. The database used were: PubMed, Virtual Health Library (VHL) and SciELO and other nutrition journals. The strategy elaborated for inclusion in this research were: a study published in journals indexed in English, Spanish and Portuguese, available in full, free of charge, addressing the proposed theme, which were duly published in the period 2015 to 2021. The strategy elaborated for exclusion were studies published before 2015, which were not available in full, and were unrelated to the proposed theme. Thus, 367 studies were found, of this total 85 were selected, but only 37 studies were analyzed in this article. It is concluded that there are different risk factors for malnutrition in the elderly, this nutritional status is a risk factor for the development of diseases and can lead to death. Therefore, family members should act to avoid this situation, but when developed it is relevant that the elderly be accompanied by the nutritionist, because the professional can analyze the clinical picture and develop appropriate dietary strategies for patients.
\end{abstract}

Keywords: Quality of life; Eating habits; Old person.

\section{Resumen}

El objetivo general del estudio es destacar la importancia de los hábitos alimenticios saludables entre los ancianos desnutridos. Se menciona que los objetivos específicos del estudio son: Comprender la relación entre los cambios fisiológicos que se producen en el envejecimiento y las necesidades nutricionales de los ancianos. plantear aspectos de la etiología de la desnutrición en las personas mayores; y destacar la importancia del seguimiento nutricional en la población anciana en el desarrollo de hábitos saludables. Esta investigación es una revisión de la base de datos integradora, utilizada fueron: PubMed, Biblioteca Virtual en Salud (BVS) y SciELO y otras revistas de nutrición. La estrategia elaborada para su inclusión en esta investigación fue: un estudio publicado en revistas indexadas en inglés, 
español y portugués, disponible en su totalidad, de forma gratuita, abordando el tema propuesto, los cuales fueron debidamente publicados en el período 2015 a 2021. La estrategia elaborada para la exclusión fueron estudios publicados antes de 2015, que no estaban disponibles en su totalidad y no estaban relacionados con el tema propuesto. Así, se encontraron 367 estudios, de este total se seleccionaron 85, pero solo se analizaron 37 estudios en este artículo. Se concluye que existen diferentes factores de riesgo para la desnutrición en los ancianos, este estado nutricional es un factor de riesgo para el desarrollo de enfermedades, y puede llevar a la muerte. Por lo tanto, los familiares deben actuar para evitar esta situación, pero cuando se desarrolle es relevante que los ancianos estén acompañados por el nutricionista, ya que el profesional puede analizar el cuadro clínico y desarrollar estrategias dietéticas adecuadas para los pacientes.

Palabras clave: Calidad de vida; Hábitos alimenticios; Persona mayor.

\section{Introdução}

Nos últimos anos, o envelhecimento da população emergiu como uma tendência demográfica tanto em países desenvolvidos quanto países em desenvolvimento. A Pesquisa Nacional por Amostra de Domicílios (PNAD Contínua) realizada em 2017 aponta que 14,6\% da população brasileira têm 60 anos ou mais de idade, correspondendo a 30,3 milhões de pessoas (Ministério da Cidadania, 2018).

No processo de envelhecimento ocorre mudanças metabólicas, fisiológicas e bioquímicos, e ocasionam a redução da funcionalidade de diversos sistemas do corpo humano, como o respiratório, muscular e ósseo, levam a um aumento com cuidados e gastos com a saúde do indivíduo. Essas alterações deixam o organismo mais suscetível a agressores extrínsecos e intrínsecos. Além disso, as mudanças tendem a ter um efeito adverso no estado nutricional dos idosos (Oliveira et al., 2020).

Então, compreende-se que a nutrição é fundamental para a manutenção do estado funcional e da qualidade de vida dos idosos, uma vez que, eles representam o grupo com maior risco de ingestão alimentar inadequada, devido às alterações inerentes ao envelhecimento a nível fisiológico ou psicossocial. Quando, possuem quadro clínico com doenças podem produzir uma modificação nos comportamentos alimentares, que influenciam a desnutrição (Oliveira et al., 2021).

Diante dessa realidade, compreende-se que os hábitos alimentares atuais da população têm grande impacto no seu estado nutricional e consequentemente na sua saúde. A Organização Mundial de Saúde (OMS) aponta que entre os dez fatores de riscos para o desenvolvimento de doenças crônicas, cinco estão intimamente relacionados à dieta e exercício físico como obesidade, estilo de vida sedentário, pressão alta, hipercolesterolemia e consumo insuficiente de frutas e vegetais (Pereira et al., 2017).

Sobre o estado nutricional dos idosos, Maciel et al. (2016), Pacheco et al. (2020) e Oliveira et al (2020) elucidam que a maioria dos idosos possui uma dieta pobre de nutrientes. Pois, não ingere fibras, minerais e vitaminas em quantidade suficiente. Então, quando a alimentação não fornece ao idoso os nutrientes necessários, sua saúde pode ser prejudicada, expondo-o a diversas doenças, por isso o acompanhamento contínuo do estado nutricional do idoso é importante.

As consequências da má nutrição incluem: fragilidade, magreza, mobilidade restrita, fragilidade da pele, maior risco de quedas, fraturas, agravamento das condições de saúde e aumento da mortalidade (Albuquerque et al., 2018). A desnutrição contribui para um declínio progressivo da saúde, colocando pacientes em risco de eventos graves de saúde. Esses eventos são os precursores do aumento da deficiência de mobilidade, redução da qualidade de vida, institucionalização prematura ou morte, em idosos independentes anteriormente saudável (Júnior et al., 2019).

Nesse sentido, a pesquisa justifica-se pela necessidade de evidenciar a importância da alimentação saudável na terceira idade com a finalidade de oferecer qualidade de vida e saúde ao idosos. Antes exposto, os hábitos alimentares corretos são oferecidos através da promoção de estratégias como acompanhamento familiar e profissional para uma alimentação saldável, que contribuirá para um envelhecimento com condições físicas, biológicas e qualidade de vida mais satisfatória.

Portanto, compreende-se que conhecer os hábitos alimentares da população idosa é importante porque influencia o estado nutricional e consequentemente auxilia na sua saúde, bem-estar e qualidade de vida. Assim, a questão problema do estudo é: qual a importância dos hábitos alimentares saudáveis na terceira idade? 
O objetivo geral do estudo é destacar a importância dos hábitos alimentares saudáveis entre idosos desnutridos. Menciona-se que os objetivos específicos do estudo são: Compreender a relação entre as alterações fisiológicas que acontecem no envelhecimento e as necessidades nutricionais do idoso. Levantar aspectos da etiologia da desnutrição em idosos; e destacar a importância do acompanhamento nutricional na população idosa no desenvolvimento de hábitos saudáveis.

Para responder à questão problemas e alcançar os objetivos proposto do estudo foi realizado uma pesquisa por meio da revisão da literatura, no qual limitou-se a estudar artigos, dissertações, livros e teses publicados entre 2016 a 2021 na área de nutrição e saúde, os dados receberam tratamento qualitativo.

\section{Metodologia}

Esta pesquisa trata-se de uma revisão da integrativa com metodologia abrangente, imparcial e reprodutível, que possuía a finalidade de responder uma questão específica. Segundo Campos (2019) as revisões integrativas fornecendo uma síntese do conhecimento disponível.

Uma das etapas da revisão é a busca da literatura que visa obter um apanhado bibliográfico sobre o tema proposto (Kleina e Rodrigues, 2014). Assim, para a construção dessa pesquisa a estratégia adotada foi encontrar estudo elegíveis encontradas em artigo, dissertação, monografias e teses.

A base de dados utilizadas foram: PubMed, Biblioteca Virtual de Saúde (BVS) e SciELO e outras revistas de nutrição. Os descritores usados para pesquisa em base de dados foram: Qualidade de Vida, Hábitos Alimentares, e Pessoa Idosa.

A estratégia elaborada para a inclusão nessa pesquisa foram: estudo publicados em revistas indexadas em inglês, espanhol e português, disponíveis na íntegra, gratuitos, que abordassem sobre o tema proposto, que foram devidamente publicados no período de 2015 a 2021. A estratégia elaborada para exclusão foram estudos publicados antes de 2015, que não estavam disponíveis na integra, e não tinham relação com o tema proposto. Assim, foram encontrados 367 estudos, desse total foram selecionados 85 , mas apenas 37 estudos foram analisados nesse artigo.

\section{Resultados e Discussão}

\subsection{Processo de envelhecimento e as necessidades nutricionais}

O processo de envelhecimento envolve mudanças que aparecem gradualmente e que têm a ver com a manutenção da qualidade de vida tais como: a diminuição do funcionamento dos órgãos ou sistemas, perda de autonomia e perda de padrões sociais relacionados a ser pessoas produtivo (Bernardi et al., 2017). A prevalência de problemas relacionados ao estado nutricional também aumenta, variando da desnutrição ao sobrepeso e obesidade (Pacheco et al., 2020).

Embora ciências e a tecnológico tenham avançado significativamente resultado em um aumento substancial na expectativa vida. Não foram levaram em consideração a uma qualidade vida dos idosos, uma vez que, convivem diariamente com doenças crônicas e deficiência motoras (Simieli et al., 2019).

Os principais indicadores de mau estado nutricional em idosos são: perda de peso, redução significativa na circunferência do braço, aumento ou diminuição das dobras cutâneas, redução significativa da albumina do soro, mudança significativa na subtração funcional, ingestão alimentar inadequada, e níveis inadequados de vitaminas, minerais ou lipídios no sangue. Assim como, a circunferência da panturrilha é um dos principais indicadores de desnutrição proteica em idosos (Da Paixão et al., 2020).

Para evitar doenças relacionadas a idade é fundamental que o idoso possua hábito saudáveis, a fim de que esse grupo permaneça independente e continuem a participar ativamente na vida da família e da comunidade (Silveira e Almeida, 2018). Assim, a promoção de saúde é a garantia de prevenir ou retardar o início de doenças crônicas como câncer, diabetes tipo II, 
acidente vascular cerebral, demência e doenças cardiovasculares, levando à incapacidade e à mortalidade relacionada (Barbosa et al., 2015).

Por esse motivo, a nutrição é vista como o fator que influencia na saúde do idoso, e deve ser levado em consideração na promoção da saúde e prevenção de doenças nesse público (Oliveira et al., 2020). para que idoso tenha as necessidades nutricionais atingidas é necessário que o profissional de nutrição realize avaliação para adequação de energia, proteínas, vitaminas e minerais (MacieL et al., 2015).

Desse modo, o acompanhamento nutricional do idosos está baseado em incorporar uma alimentação saudável e adaptada, quando considerado as mudanças fisiológicas que ocorrem devido ao processo de envelhecimento. Assim sendo, o acanhamento nutricional tem a finalidade de proporcionar uma melhor qualidade de vida ao indivíduo onde ele possa se adaptar à sua nova condição (Aires et al., 2019).

\subsection{Necessidades dietéticas especiais para idosos}

Em relação à literatura, foi possível identificar alguns dos componentes essenciais da dieta alimentar do idoso, como líquidos, fibra, redução de sódio, redução de açúcar e vitamina D (Alvarado-García et al., 2017).

A ingestão hídrica diária recomendada para idosos é de aproximadamente $30 \mathrm{ml} / \mathrm{kg}$ de peso corporal, um mínimo de $1.000 \mathrm{ml}$ de líquidos é necessário para compensar exclusivamente as perdas insensíveis. A hidratação é uma das necessidades mais importantes do idoso devido à redução da sensação de sede e ao consumo de certos medicamentos, como os diuréticos, que alteram esse equilíbrio (Guimarães et al., 2021).

O consumo de fibras entre os idosos é baixo na maioria das vezes, devido à diminuição do consumo de frutas e vegetais por diversos fatores. É o nutricionista orientar o consumo de fibra regularmente. A adição de fibra à dieta tem sido associada à diminuição do açúcar e da gordura no sangue, estimulação do peristaltismo e da função gastrointestinal e redução do peso, pois promove saciedade em menores níveis calóricos e lipídicos (Oliveira et al., 2019).

Algumas condições de saúde relacionadas ao envelhecimento, como hipertensão, risco de acidente vascular cerebral, hipertrofia ventricular esquerda e proteinúria, são contrabalançadas pela diminuição do consumo de sal / sódio. Para conseguir essa redução, é necessário conhecer os alimentos que devem ser evitados (Pereira et al, 2018).

Devido à diminuição da secreção de enzimas pelo pâncreas e diminuição dos receptores de insulina durante o envelhecimento, os idosos são mais propensos ao diabetes mellitus. Por isso, nessa população, o consumo de açúcar deve ser controlado, não ultrapassado os $10 \%$ do consumo total de carboidratos. Então, os açúcares refinados presentes em bolos, tortas e produtos processados devem ser evitados (Monteiro, 2017).

Os adultos mais velhos correm o risco de ter deficiências de vitamina $\mathrm{D}$ devido à exposição limitada à luz solar e à redução na capacidade da pele de produzir vitamina D. Em contrapartida, um terço das necessidades de vitamina D pode ser obtido com a dieta se os seguintes alimentos forem incluídos: salmão, frutos do mar frescos, sardinha ou atum em azeite, óleo de fígado de bacalhau, ovos, entre outros (Lima et al., 2019).

A vitamina D é essencial para promover a absorção do cálcio, pois promove a saúde óssea; junto com o cálcio, são os componentes mais importantes da dieta como protetores contra a osteoporose. Ele também tem um papel de auxiliar no funcionamento do sistema imunológico, secreção de insulina, função cardíaca, regulação da pressão arterial e função cerebral (Santos et al., 2019).

\subsection{Etiologia da desnutrição em idosos}

O estado nutricional é consequência do equilíbrio da ingestão de nutrientes e do gasto calórico de proteína para proteger as necessidades fisiológicas ideais; as anomalias desta condição são refletidas como desnutrição (Morais et al, 2021). A 
desnutrição pode ser considerada uma síndrome geriátrica, uma vez que favorece o aparecimento de outras doenças e aumento de infecções, pois ocorre a diminuição da imunidade. Assim, como é considera um fator de morbidade e mortalidade nesta faixa etária (Lourdes et al., 2021).

A desnutrição é definida como a condição patológica resultante de uma dieta insuficiente ou má assimilação de alimentos, relacionada à perda de peso e massa muscular, diminuição da força e imunodeficiência. Muitas vezes, é subestimada, mas sua importância é fundamental, pois aumenta a morbimortalidade, as reinternações ou o tempo de internação e os custos com saúde (Da Paixão et al., 2020).

Nas condições de desnutrição em idosos, a deficiência de micronutrientes como cálcio, vitamina D, vitamina B12 e folato pode induzir uma diminuição na reação do sistema imunológico e causar risco de vida, por esses motivos, avaliação nutricional eficaz é a principal ferramenta utilizada para identificar os idosos em risco de desnutrição, o que daria o primeiro passo para prevenir ou restaurar o estado nutricional e restaurar a qualidade de vida (Alvarado-García et al., 2017).

A desnutrição em populações idosas é o resultado de uma combinação de variáveis interdependentes, incluindo mudanças fisiológicas, sobre isso, Albuquerque 2018) explica que gradualmente, com a idade, há uma diminuição da percepção sensorial que pode afetar o olfato, a visão e o paladar o que, consequentemente, reduz sensações de prazer e bem-estar ao se alimentar. Além disso, os distúrbios do olfato resultam em diminuição da sensibilidade olfativa e diminuição da capacidade de identificar odores agradáveis

Com o envelhecimento podem surgir uma série de modificações como a diminuição da motilidade intestinal, que favorece a constipação o que por sua vez pode levar ao aparecimento diarreicas, com a consequente diminuição na absorção de nutrientes. Surgindo, assim a importância de orientar os idosos os hábitos dietéticos que favoreçam o trânsito intestinal adequado (De Sousa Albuquerque, Cavalcante, 2016).

Declínio sociais e psicológicos contribui para aumentar o risco de desnutrição em idoso, pois a falta de estímulos de parentes e familiares, leva, em inúmeras ocasiões, à perda de interesse pela comida, com o consequente risco nutricional; esse fato pode ser agravado ainda mais no caso de idosos que moram sozinhos e daqueles que sofrem de tendências depressivas (Morais et al, 2021).

A oferta de alimentação adequada aos idosos às vezes é limitada por diversos fatores como diminuição da capacidade física para fazer compras, superação de obstáculos de moradia ou transporte, a diminuição do poder de compra, a falta de informações e conselhos para alcançar dietas balanceadas ou terapêuticas agradáveis e acessíveis, etc. (Melo e Medeiros, 2020). As diminuições na capacidade de realizar as atividades da vida diária costumam afetar o manuseio e o preparo dos alimentos, bem como o manuseio de alguns pratos e são fatores que contribuem para o consumo de dietas restritivas ou desequilibradas (Oliveira et al., 2021).

\subsection{A importância dos hábitos alimentares saudáveis em idosos desnutridos}

Como exposto ao longo do estudo, o envelhecimento pode ser definido como um processo dinâmico, gradual, natural e inevitável, no qual ocorrem mudanças a nível biológico, físico, psicológico e social, que têm um impacto inegável no estado funcional e físico das pessoas, o que também ocorre ao longo do tempo (Barbosa e Fernandes, 2020).

As intervenções nutricionais que visam reduzir a desnutrição, a fragilidade e o risco de queda nesta faixa etária estão centradas em alcançar uma nutrição adequada e corrigir quaisquer deficiências nutricionais (RODRIGUES, 2018). As recomendações para isso devem levar em consideração as barreiras e os recursos do indivíduo, além de outros dados de avaliação médica relacionados (Esquivel, 2018).

Para uma dieta equilibrada é necessária uma avaliação nutricional, pois é um processo que fornece informações necessárias para determinar o diagnóstico de subnutrição e a etiologia por trás dela e, portanto, esta etapa é a base do processo 
de cuidado nutricional. Sendo ainda mais fundamental em idosos desnutridos, pois sua causa é multifatorial (Graciano et al., 2018). No entanto, todos os profissionais de saúde devem observar que um diagnóstico nutricional não depende apenas de um único achado da avaliação nutricional, sendo fundamental a utilização de diferentes achados clínicos (Silvério et al. 2017).

Hábitos saudáveis são essenciais em todas as idades, principalmente no envelhecimento da população, pois com a idade, aumenta a probabilidade de sofrer doenças com impacto na saúde. Por isso, hábitos alimentares saudáveis e a atividade física ajudam a prevenir ou retardar doenças não transmissíveis e outras condições ao longo da vida (Jobim e Jobim, 2015). Assim, uma dieta personalizada e adaptada combinada com um programa de exercícios multicomponente é eficaz para melhorar a composição corporal e os parâmetros bioquímicos em idosos (Caldas et al., 2019).

Para garantir que os idosos possuam uma qualidade de vida é essencial que essa população tenha suas necessidades nutricionais alcançadas. Uma das estratégias para promoção de envelhecimento saudável é a Atenção Nutricional (AN), na qual o profissional de nutrição é responsável por realizar atendimento individuas e visitas domiciliares com a finalidade de garantir que o idoso tenha seus requisitos nutricionais, conforme seu quadro e histórico clínico, oferecido (Melo e Medeiros, 2020).

Além do mais, os cuidadores domiciliares e familiares estão devem possuir conhecimento para monitorar a ingestão alimentar e o estado nutricional de idosos; auxiliar em muitas tarefas relacionadas à alimentação, como obtenção e preparação de refeições, e auxiliar na alimentação quando necessário; e atuar como um canal entre o destinatário do cuidado e os profissionais de nutrição formais, como nutricionistas (Andrade et al., 2021).

Alimentação adequada é fundamental, entretanto prática de atividade física regular não pode ser esquecida, pois influenciam um envelhecimento saudável (Aires et al., 2019). Então alinhar alimentação com atividade física oferece qualidade de vida, autonomia e independência as pessoas da terceira idade. Essas atitudes são determinantes para que a população idosa tenha cada vez mais um envelhecimento melhor e mais saudável (De Sousa Albuquerque et al., 2016; Graciano et al., 2018).

Por fim, esclarece que a conscientização sobre a desnutrição entre os idosos é fundamental para o cuidado, pois influencia as políticas de atenção nutricional desse grupo. Assim, a obtenção de dados mais recentes sobre a prevalência de desnutrição entre idosos é sempre importante, pois acredita-se que isso não apenas melhorará o resultado, mas também garantirá um atendimento contínuo e evitará a deterioração progressiva do estado nutricional (Abd Aziz et al., 2017).

\section{Considerações Finais}

O estudo teve como objetivo destacar a importância dos hábitos alimentares saudáveis entre idosos desnutridos. Conclui-se que o papel da nutrição na velhice é vital. Porém, existem inúmeros processos que influenciam a redução das proteínas da dieta, como falta de apetite, problemas orais ou perda do paladar, modificações metabólicas e dificuldade de mobilidade. Então, é fundamental minimizar o risco da ingestão alimentar inadequada, seja por meio de suplementação, modificações dietéticas ou uma combinação de ambos.

O presente estudo confirma que existem diferentes fatores de risco para a desnutrição em idosos, esse estado nutricional é um fator de risco para o desenvolvimento de doenças, podendo levá-lo até a morte. Por isso, familiares devem atuar para evitar tal quadro, mas quando desenvolvidos é relevante que o idoso seja acompanhado pelo nutricionista, pois o profissional pode analisar o quadro clínico e desenvolver estratégias alimentares adequadas aos pacientes.

Além do mais, para garantir que os idosos apresentem qualidade de vida, é essencial que essa população tenha suas necessidades nutricionais alcançadas e mantenham hábitos de vidas saudáveis. Assim, surge o papel da família nos cuidados dos idosos e o do nutricionista que atua com orientados e recomendações para que essa população consiga manter-se com uma alimentação adequada, adotando um estilo de vida mais saudável. A atividade física, também, deve ser realizada pelos idosos, porém é importante destacar que as atividades físicas sempre são possíveis, principalmente em idosos desnutridos, pois muitas vezes não possuem mobilidade. 
Para estudo futuros sugere-se pesquisa sobre a importância dos hábitos saudáveis em instituições de longa permanência, pois nesses locais os idosos, muito vezes, não contam com o cuidado de familiares e por isso estão sujeitos ao desenvolvimento de outros distúrbios alimentares. Entretanto, destaca-se que o estudo possui limitações, pois limitou-se em estudados hábitos alimentares saudáveis em idosos desnutridos, porém essa população pode estar obsessa, sendo esse em quadro que necessita de acompanhamento nutricional.

\section{Referências}

Abd Aziz, N. A. S., Teng, N. I. M. F., Hamid, M. R. A., \& Ismail, N. H. (2017). Assessing the nutritional status of hospitalized elderly. Clinical interventions in aging, 12, 1615 .

Andrade, C. C. S., Andrade, C. H. S., \& de Almeida Andrade, E. (2021). A importância da assistência de enfermagem e nutrição na prevenção de quedas em idosos. Revista Artigos. Com, 30, e8129-e8129.

Albuquerque, Samara Maria de et al. (2018) Fatores que afetam o consumo alimentar e a nutrição do idoso: uma revisão integrativa. Trabalho de Conclusão de Curso (Bacharel em Nutrição) - Unidade Acadêmica de Saúde da Universidade Federal

Alvarado-García, A., Lamprea-Reyes, L., \& Murcia-Tabares, K. (2017). Nutrição na pessoa idosa: uma oportunidade para o cuidado de enfermagem. Enfermagem da Universidade, 14 (3), 199-206.

Alvarado Gutiérrez, M. C. (2019). Hábitos alimentarios y estado nutricional en adultos mayores Centro de Salud Pachacutec Cajamarca. Disertación (Licenciada en Enfermería) Universidad Nacional De Cajamarca - UNC.

Aires, I. O., de Sousa, L. L. C., de Sousa, D. J. M., de Araújo, D. S. C., Oliveira, I. K. F., \& Alencar, M. D. S. S. (2019). Consumo alimentar, estilo de vida e sua influência no processo de envelhecimento. Research, Society and Development, $8(11)$.

Barbosa, K. T. F., \& Fernandes, M. D. G. M. (2020). Vulnerabilidade da pessoa idosa: desenvolvimento de conceito. Revista Brasileira de Enfermagem, 73.

Barbosa, L. M., Czernaik, C. M., \& Colussi, E. L. (2015). Nutrição e aspectos sociais no envelhecimento. Telma Elita Bertolin. Méritos

Bernardi, A. P., Maciel, M. A., \& Baratto, I. (2017). Educação nutricional e alimentação saudável para alunos da Universidade Aberta a Terceira Idade (UNATI). RBONE-Revista Brasileira de Obesidade, Nutrição e Emagrecimento, 11(64), 224-231.

Campos, J. M. (2019). Manual Prático de Pesquisa Científica. Thieme Revinter Publicações LTDA.

Caldas, L. R. D. R., Albuquerque, M. R., Araújo, S. R. D., Lopes, E., Moreira, A. C., Cândido, T. M., \& Carneiro-Júnior, M. A. (2019). Dezesseis semanas de treinamento físico multicomponente melhoram a resistência muscular, agilidade e equilíbrio dinâmico em idosas. Revista brasileira de ciências do esporte, 41 , $150-156$.

da Paixão, A. A., Ximenes, L. D. S. V., \& dos Santos, E. T. (2020). Tendências temporais da mortalidade por desnutrição em idosos no estado de mato grosso do sul, no período de 2002 a 2012. Revista Eletrônica da Associação dos Geógrafos Brasileiros Seção Três Lagoas, 48-65.

de Sousa Albuquerque, F. R. V., \& Cavalcante, J. L. P. (2016). Constipação intestinal e consumo alimentar em grupo de idosos: um estudo bibliográfico. Revista Kairós: Gerontologia, 19(4), 293-304.

Esquivel, M. K. (2018). Nutritional assessment and intervention to prevent and treat malnutrition for fall risk reduction in elderly populations. American journal of lifestyle medicine, 12(2), 107-112.

Graciano, A. R., Cozer, A. M., Santana, V. M. L., \& de Oliveira, J. M. R. (2018). Avaliação nutricional e risco de desnutrição em idosos com demências. Saúde e Pesquisa, 11(2), 293-298.

Guimarães, B. P., Maciel, A. R., Barbosa, A. P., Viziolli, B. N. P., Vitta, C. E., \& Chaud, D. M. A. (2021). O consumo de água em idosos: uma revisão. Vita et Sanitas, v. 15, n. 2, p. 53-69, 2021.

Jobim, F. A. R. D. C., \& Jobim, E. F. D. C. (2015). Atividade física, nutrição e estilo de vida no envelhecimento. UNOPAR Cient., Ciênc. biol. saude.

Júnior, E. O. C., de Araújo, E. Q. X., Evangelista, D. R., Rezende, F. A. C., Netto, L. S. S., Osório, N. B., \& Nunes, D. P. (2019). Relação das condições de vida e saúde sobre a fragilidade em idosos. Humanidades \& Inovação, 6(11), 76-87.

Kleina, C., \& Rodrigues, K. S. B. (2014). Metodologia da pesquisa e do trabalho científico. Ed. Curitiba, PR: IESDE Brasil.

Lima, E. F. C., Formiga, L. M. F., Costa, D. M., Feitosa, L. M. H., Araújo, A. K. S., \& da Rocha Leal, S. (2019). Ingestão alimentar de cálcio e vitamina D em idosos. Revista Enfermagem Atual In Derme, 87(25).

Maria de Lourdes, M. V., Leonor, H. P., \& Maritza, C. S. (2021). La Nutrición En El Adulto Mayor, Un Reto Social. In: cibamanz.

Maciel, B. K. C., Fortunato, B. E. N., Melo, L. F. B., \& Dantas, S. R. (2015). Necessidades Nutricionais: mudanças com o envelhecimento. In Congresso Internacional de Envelhecimento Humano.

Ministério Da Cidadania (Secretaria Especial do Desenvolvimento Social). Estratégia Brasil Amigo da Pessoa Idosa. Acesso em: 19 de set de 2021. 
Research, Society and Development, v. 10, n. 14, e203101421858, 2021

(CC BY 4.0) | ISSN 2525-3409 | DOI: http://dx.doi.org/10.33448/rsd-v10i14.21858

Melo, C. L. D., \& Medeiros, M. A. T. D. (2020). Atenção Nutricional à pessoa idosa na Atenção Primária à Saúde, sob a ótica de profissionais de saúde. Revista Brasileira de Geriatria e Gerontologia, 23.

Morais, S. C. M., Santos, D. A., de Almeida, E. B., \& da Silva, T. B. L. (2021). Aspectos nutricionais e psicológicos de idosos em situação de vulnerabilidade: Um estudo de revisão sistemática. Revista Kairós: Gerontologia, 24, 157-176.

Monteiro, M. D. S. Autocuidado praticado por idosos com diabetes mellitus em uma unidade básica de saúde-Parintins-AM. Trabalho de Conclusão de enfermagem (Enfermagem) - Universidade do Estado do Amazonas.

Oliveira, A. S., de Oliveira Santos, J., Gonçalves, I. C. M., \& Soares, W. D. (2021). Prevalência da desnutrição em idosos. Revista Perspectiva, 45(169), 141154.

Oliveira, L. M. C. D., Dantas, M. L. D. S., \& Feitosa Neto, P. A. (2021). Qualidade de vida e capacidade funcional segundo estado nutricional de idosos em Maceió, AL. Trabalho de conclusão de curso (Bacharel em Nutrição) - Centro Universitário Tiradentes.

Oliveira, M. D. D., Cas, E. M. D., Tassoni, D. S., Ziemann, D. K., Poll, F. A., Wichmann, F. M. A., \& Roos, N. P. (2019). Con sumo alimentar de fibras e caracterização do perfil nutricional de idosos hipertensos. Anais do Salão de Ensino e de Extensão, 93.

Pacheco, R., Silva, R., Costa, T., Almeida, A., \& Amado, J. (2020). Fatores de risco de desnutrição na pessoa idosa: uma revisão sistemática. Millenium-Journal of Education, Technologies, and Health, (13), 69-78.

Pereira, H. E. F., Oliveira, J. S., Prates, R. P., Leão, L. L., Pereira, É. J., \& Farias, P. K. S. (2018). Perfil nutricional e dietético de idosos atendidos nas estratégias de saúde da família do norte de Minas Gerais. Revista de APS, 2l(2).

Rodrigues, R. M. D. S. N. (2018). Abordagem da educação alimentar e nutricional no contexto escolar através do Parâmetros Curriculares Nacionais (PCNs) transversal de saúde: um estudo de caso. Revista Científica de Iniciación a la Investigación, 3(1).

Santos, J. V. D., Closs, V. E., Castanho, V. C., \& Hagen, M. E. K. (2019). Deficiência de vitamina D nos idosos brasileiros e fatores associados: uma revisão sistemática (resultados parciais). Clinical and biomedical research.

Simieli, I., Padilha, L. A. R., \& de Freitas Tavares, C. F. (2019). Realidade do envelhecimento populacional frente às doenças crônicas não transmissíveis. Revista Eletrônica Acervo Saúde, (37), e1511-e1511.

Silveira, J. R. D., \& Almeida, S. G. D. (2018). Alimentação do idoso: Estratégia de motivação para uma alimentação saudável. Trabalho de conclusão de curso (Nutrição) - Centro Universitário De Brasília (UNICEUB), 2018.

Silvério, J. K. A., Pedreira, K. R. A., \& Kutz, N. A. (2017). Estado nutricional de idosos institucionalizados: uma revisão de literatura. Visão Acadêmica, 17(3).

Sousa, K. T. D., Mesquita, L. A. S. D., Pereira, L. A., \& Azeredo, C. M. (2014). Baixo peso e dependência funcional em idosos institucionalizados de Uberlândia (MG), Brasil. Ciência \& Saúde Coletiva, 19, 3513-3520. 